USTC-ICTS-11-14

\title{
Strings from geometric tachyon in Rindler space and black hole thermodynamics
}

\author{
Huiquan Li 1 \\ Interdisciplinary Center for Theoretical Study \\ University of Science and Technology of China, Hefei, Anhui 230026, China
}

\begin{abstract}
The dynamics of a probe particle or wrapped brane moving in the two-dimensional Rindler space can be described by a time-dependent tachyon field theory. Using knowledge of tachyon condensation, we learn that the infalling brane gets thermalised and produces open string pairs at the Hagedorn temperature when entering into the near-horizon Rindler wedge. It is shown that the Hagedorn temperature of the infalling brane is equal to the Hawking temperature of the host black hole detected in the same time coordinate. The infalling brane will decay completely into closed strings, mainly massive modes, when it reaches the horizon in infinitely long time as observed by observers at spatial infinity. Preliminary estimates indicate that the degeneracy of states of the closed strings emitted from the infalling brane should be responsible for the increased entropy in the host black hole due to absorption of the brane.
\end{abstract}

\section{Introduction}

String theory as a quantum gravity has been proven successful in providing microscopic interpretation to black hole thermodynamics. The Bekeinstein-Hawking entropy is reproduced in some extremal D-branes by counting the underlying string states [1]. The perfect agreements are further found by extending to the case of near-extremal black branes with small temperature [2, 3], which can be taken as a linear excitation above the ground state

\footnotetext{
${ }^{1}$ E-mail: hqli@ustc.edu.cn
} 
of extremal branes. The Hawking radiation accounting for the temperature is interpreted as open strings colliding to forming massless closed strings [2].

However, it is hard to go beyond to study the generical non-extremal black holes or branes away from extremality. For these black holes, the near-horizon geometry is the Rindler space. The linear perturbative analysis does not apply because of strong backreactions. In this case, the relevance of the thermodynamics to closed string tachyon has been realised in [4, 5].

In this work, we try to explore the stringy understanding of the thermodynamics of non-extremal black holes or branes by dropping in probe branes, since a large black hole can be formed by swallowing material repeatedly starting from a small one. The study of the infalling process of material is directly relevant to the information loss problem [6]. Moreover, probe matter branes are also widely adopted in holographic models of QCD, condensed matter and other topics at finite temperature.

We show that a probe particle or wrapped brane moving in the near-horizon Rindler space can be described by the time-dependent tachyon field theory. So, from the effective theory or the boundary conformal field theory (BCFT) [7, 8, 9] of rolling tachyon, we learn that the probe brane will experience a Hagedorn transition when it falls into the Rindler wedge. Open strings [10, 11, 12] and closed strings [13, 14] will be produced from the infalling brane at the Hagedorn temperature, which is equal to the Hawking temperature of the host black hole. The infalling brane will decay completely into closed strings as the horizon is reached. The degeneracy of states of the emitted closed strings contributes the same order of entropy increased in the host black hole due to absorption of the brane.

We first present the tachyon field configurations that describe the dynamics of a probe moving in near-horizon Rindler space in Section. 2. In Section. 3, we introduce the results on string production from the rolling tachyon obtained in the corresponding BCFT and explore their implications to black hole thermodynamics. Conclusions are made in the last section. In this paper, we adopt the unit $\alpha^{\prime}=1$.

\section{Geometrical tachyon in Rindler space}

For non-extremal black holes or black p-branes, the geometry near the event horizon is generically Rindler space in the time and radial directions. In this section, we take two examples to demonstrate that a probe particle or wrapped $q$-brane moving in this spacetime can be described by a time-dipendent tachyon field theory. 


\subsection{Schwarzschild black hole}

We start with the simple and well studied case, the Schwarzschild black hole:

$$
d s^{2}=-\left(1-\frac{r_{0}}{r}\right) d t^{2}+\left(1-\frac{r_{0}}{r}\right)^{-1} d r^{2}+r^{2} d \Omega_{2}^{2},
$$

where $r_{0}=2 M$ and $M$ is the mass of the black hole. The Hawking temperature and the entropy are respectively

$$
T_{H}=\frac{1}{8 \pi M}, \quad S_{B H}=\frac{A}{4}=4 \pi M^{2} .
$$

The Hawking radiation can be derived by introducing a scalar $\phi$ with mass $m$ (a simple situation is $m=0$ ) in the black hole background. Defining the tortoise coordinate $r_{*}=$ $r+r_{0} \ln \left(r / r_{0}-1\right)$ and separating out the angular components, one obtains the following Schrödinger equation:

$$
\left[\partial_{t}^{2}-\partial_{r_{*}}^{2}+W\left(r_{*}\right)\right] \phi\left(r_{*}, t\right)=0,
$$

where $W\left(r_{*}\right)=\left(1-r_{0} / r\right)\left[r_{0} / r^{3}+l(l+1) / r^{2}+m^{2}\right]$. Approaching the horizon $r \rightarrow r_{0}$, $r_{*} \rightarrow-\infty$ and $W \sim e^{r_{*} / r_{0}}$. Thus, the metric near horizon is approximately: $d s_{2}^{2} \simeq$ $e^{r_{*} / r_{0}}\left(-d t^{2}+d r_{*}^{2}\right)$. For $r \rightarrow \infty, r_{*} \rightarrow \infty$ and $W \rightarrow l(l+1) / r_{*}^{2}$ in the massless case.

The near horizon geometry along the time and radial directions is the Rindler space: $d s_{2}^{2}=-\rho^{2} d t^{2}+4 r_{0}^{2} d \rho^{2}$, with the coordinate redefinition $r=r_{0}\left(1+\rho^{2}\right)$ ( $\rho$ is dimensionless $)$. The Hawking temperature $T_{H}$ given in Eq. (2) arises from the periodicity of the Euclidian time coordinate: $t /\left(2 r_{0}\right) \rightarrow t /\left(2 r_{0}\right)+2 \pi i$. The dynamics of a test particle moving towards the horizon along the radial direction is governed by

$$
S_{0}=-m_{0} \int d t \sqrt{\rho^{2}-4 r_{0}^{2}\left(\partial_{t} \rho\right)^{2}}=-\int d x^{0} V(T) \sqrt{1-\dot{T}^{2}},
$$

where

$$
x^{0}=\frac{t}{r_{0}}, \quad T=-2 \ln \rho, \quad V(T)=r_{0} m_{0} e^{-T / 2} .
$$

We denote $\dot{T}=\partial_{x^{0}} T$. In the $x^{0}$ coordinate, the Hawking temperature becomes $1 /(4 \pi)$ due to $x^{0} / 2 \rightarrow x^{0} / 2+2 \pi i$. Approaching the horizon $\rho \rightarrow 0$, we have $T \rightarrow \infty$ and $V(T) \rightarrow$ 0 . This action is the tachyon field action with the potential: $V(T)=T_{0} / \cosh (T / 2)$ $\left(T_{0}=m_{0} r_{0} / 2\right)$ at late times when the tachyon field $T$ is large [9]. The latter is derived in open string field theory [15]. It describes the decaying process of an unstable D-particle in bosonic string theory. Such a construction of tachyon field theory is similar to the spirit of the geometrical tachyon configuration near NS5-branes [16, 17]. The tachyonic instability of D0-brane probing Shcwarzschild horizon was also noticed in [18]. 
If there is no energy loss from the rolling tachyon, the energy density should be constant

$$
T_{00}=\frac{V(T)}{\sqrt{1-\dot{T}^{2}}}=C,
$$

which gives $\ddot{T}=V^{2} /\left(2 C^{2}\right)$. Since we are focusing on the dynamics near horizon and do not know the information before it entering into the Rindler space, we can take the constant $C$ as the energy density when the particle enters the Rindler space. In the nearhorizon region, the infalling particle behaves like an unstable particle at late times with large $T$. So the solution is approximately: $T \simeq x^{0}+a e^{-x^{0}}+\cdots$, with $a=\left(r_{0} m_{0} /(\sqrt{2} C)\right)^{2}$. The pressure is

$$
T_{i j}=-\frac{V^{2}}{C} \delta_{i j} \simeq-\frac{r_{0}^{2} m_{0}^{2}}{C} e^{-x^{0}} \delta_{i j} .
$$

Thus, the tachyon condensation leads to a pressureless state approaching the horizon, which corresponds to non-relativistic, massive closed strings [19, 9]. This will be addressed in the next section.

\section{$2.2 \quad$ Black branes}

We next consider the black brane gravitational solutions [20, 21] in type II string theories. Redefine the coordinate $r^{7-p}=\left(1+\rho^{2}\right) r_{+}^{7-p}$ so that the event horizon is located at $\rho=0$. In non-extremal case, the black $p$-brane $(p<7)$ metric near the event horizon takes the form

$$
\begin{gathered}
d s^{2}=\epsilon^{-\frac{7-p}{2}}\left[-\rho^{2} d t^{2}+\epsilon^{7-p} d \vec{x}^{2}+\frac{4 r_{+}^{2}}{(7-p)^{2}} \epsilon^{p-5} d \rho^{2}+r_{+}^{2} \epsilon^{2} d \Omega_{8-p}^{2}\right], \\
e^{\Phi}=g_{s} \epsilon^{\frac{(p-3)(7-p)}{4}} .
\end{gathered}
$$

where the parameter $\epsilon$ is defined as the discrepancy between the radii $r_{ \pm}$of the inner and outer horizons: $\epsilon^{7-p}=1-\left(r_{-} / r_{+}\right)^{7-p}$. The radii $r_{ \pm}$are related to the tension and the RR charge of black branes. The extremal limit is $\epsilon \rightarrow 0$ with $r_{-}=r_{+}$, in which the tension and charge are equal. When the black brane is chargeless, $r_{-}=0$ and so $\epsilon=1$.

A D-particle or $\mathrm{D} q$-brane wrapped on circles moving along the radial direction is described by the action:

$$
S_{q}=-\tau_{q} \int d t e^{-\Phi} \sqrt{-\operatorname{det} G}=\int d x^{0} V(T) \sqrt{1-\dot{T}^{2}},
$$

where

$$
T=-\sqrt{2} \ln \rho, \quad x^{0}=\frac{7-p}{\sqrt{2} r_{+}} \epsilon^{\frac{5-p}{2}} t,
$$




$$
V(T)=2 T_{q} e^{-T / \sqrt{2}}, \quad T_{q}=\frac{r_{+}}{\sqrt{2}(7-p)} g_{s}^{-1} \tau_{q} \epsilon^{\frac{p^{2}-7 p+4}{4}} .
$$

The Hawking temperature of the host black brane (8) detected in the $x^{0}$ coordinate is $1 /(2 \sqrt{2} \pi)$ due to the periodicity $x^{0} / \sqrt{2} \rightarrow x^{0} / \sqrt{2}+2 \pi i$ in the Rindler space: $d s_{2}^{2}=$ (const.) $\left[-\rho^{2}\left(d x^{0}\right)^{2}+2 d \rho^{2}\right]$. The tachyon potential $V(T)$ is the large $T$ case of the potential in superstring theory: $V(T)=T_{q} / \cosh (T / \sqrt{2})$ obtained in [15]. It is constructed like this because we are discussing the dynamics in the black brane background (8) obtained in type II superstring theories.

In this action, the non-vanishing energy-momentum tensor at late times evolves as

$$
T_{00}=C, \quad T_{i j} \simeq \frac{4 T_{q}^{2}}{C} e^{-\sqrt{2} x^{0}} \delta_{i j}
$$

with the tachyon solution: $T \simeq x^{0}+a e^{-\sqrt{2} x^{0}}+\cdots$, where $a=\sqrt{2}\left(T_{q} / C\right)^{2}$. We also get pressureless matter towards the end of condensation as $x^{0} \rightarrow \infty$.

\section{Strings from tachyon and black hole thermody- namics}

It is known that the tachyon effective action with the potential $V(T)=T_{q} / \cosh (\beta T)$ ( $\beta=1 / 2$ for bosonic string and $=1 / \sqrt{2}$ for superstring) has a corresponding $c=1 \mathrm{BCFT}$ description [22, 14, 9]. The BCFT worldsheet action is [7, 8]

$$
S=-\frac{1}{2 \pi} \int_{\Sigma} d^{2} z \partial X^{\mu} \bar{\partial} X_{\mu}+\int_{\partial \Sigma} d \tau m^{2}\left(X^{0}(\tau)\right)
$$

where $\tau$ labels the coordinate on the boundary of the worldsheet. In bosonic string theory, the boundary interaction term $m^{2}\left(X^{0}\right)$ is $(\lambda / 2) e^{X^{0}}$ (for $C=T_{q}$ ) or $\lambda \cosh X^{0}$ (for general $C)$. The latter gives rise to a full S-brane, which describes the creation and then decaying processes of an unstable D-brane, while the former gives rise to a half S-brane, which describes the decaying process of an unstable D-brane. In superstring theory, the tachyon profile takes the form: $\lambda \cosh \left(X^{0} / \sqrt{2}\right)$.

In order to understand the near-horizon behaviour of a probe brane discussed in the previous section, we only need to concentrate on the late-time evolution of the rolling tachyon. The non-vanishing components of the energy-momentum tensor at late times obtained from evaluation on the $X^{0}$ component of the boundary states are given by (in the $g_{s} \rightarrow 0$ limit) [7, 8]:

$$
T_{00}=\frac{1}{2} T_{q}[1+\cos (2 \lambda \pi)], \quad T_{i j} \simeq \begin{cases}-\frac{1}{\sin (\lambda \pi)} T_{q} e^{-x^{0}} \delta_{i j}, & \text { (bosonic string) } \\ -\frac{1}{\sin ^{2}(\lambda \pi)} T_{q} e^{-\sqrt{2} x^{0}} \delta_{i j}, & \text { (superstring) }\end{cases}
$$


where $T_{q}$ is the tension of the unstable $\mathrm{D} q$-brane and $0 \leq i, j \leq q$. Hence, the tachyon BCFT (14) is comparable to the tachyon effective actions constructed in the time coordinate $x^{0}$ in the previous section. The string production from decaying tachyon based on the BCFT has been studied previously. In what follows, we investigate the implications of the results to the thermodynamics of non-extremal black holes.

\subsection{Open string pair creation: Hagedorn and Hawking temper- atures}

The creation of open string pairs from the rolling tachyon with non-vanishing $g_{s}$ is discussed in [10, 11, 12] in the context of S-branes. In their treatments, a minisuperspace approximation is adopted, i.e., only zero modes $x^{\mu}=(\hat{t}, \vec{x})$ of $X^{\mu}=\left(X^{0}, \vec{X}\right)$ are considered. If assuming $x^{\mu}$ to be independent of the worldsheet coordinate $\sigma$, we can obtain an effective action from the action (14), from which the Hamiltonian can be derived. We thus can get the Klein-Gordon equation for the wave function $\psi(t, \vec{x})$ of free open strings by making the constraint $L_{0}+\widetilde{L}_{0}=0$ :

$$
\left[\partial_{\hat{t}}^{2}-\vec{\nabla}_{\vec{x}}^{2}+2 m^{2}(\hat{t})+(N-1)\right] \psi(\hat{t}, \vec{x})=0,
$$

where $N$ is the contribution from oscillators. This equation with exponentially increasing $m^{2}(\hat{t})$ is similar to Eq. (3) in the near-horizon region with $W \sim e^{r_{*} / r_{0}}$. The wave function can be expanded in the way: $\psi(\hat{t}, \vec{x})=u(\hat{t}) e^{i \vec{p} \cdot \vec{x}}$. The calculation of the in and out states indicates that open string pairs are created at $\hat{t} \rightarrow \infty$ stating from a vacuum with no incoming open strings at $\hat{t} \rightarrow-\infty$. The energy spectrum of the created open strings is thermal with the Hagedorn temperature, which we denote as $T_{\text {Hag }}^{\left(x^{0}\right)}$, since this temperature is derived in the BCFT or the field theories (41) and (10) constructed in the $x^{0}$ coordinate. For observers at spatial infinity, the Hagedorn temperature should be the one observed in the black hole coordinate $t$. We denote the temperature in this frame as: $T_{\text {Hag }}^{(t)}=T_{\text {Hag }}^{\left(x^{0}\right)}\left(\partial x^{0} / \partial t\right)$.

For bosonic string case, the open strings are created at the Hagedorn temperature $T_{\text {Hag }}^{\left(x^{0}\right)}=1 /(4 \pi)$. As stated in Section. 2.1, this temperature is also the Hawking temperature detected in the $x^{0}$ coordinate. Therefore, the Hagedorn temperature for open string creation on the infalling particle in the $t$ coordinate is exactly the Hawking temperature $T_{H}$ (also detected in $t$ ) of the host black hole. In summary, the relation between the two temperatures can be expressed as follows

$$
T_{H}=T_{\text {Hag }}^{(t)}=\frac{1}{r_{0}} T_{\text {Hag }}^{\left(x^{0}\right)}, \quad T_{\text {Hag }}^{\left(x^{0}\right)}=\frac{1}{4 \pi},
$$


where $T_{H}$ is given in Eq. (21) for Schwarzschild black hole. This means that the infalling material gets thermalised at the Hagedorn temperature or the Hawking temperature when it enters into the near-horizon Rindler space.

As noted in 12, this Hagedorn temperature for open string creation essentially arises from the periodicity of $X^{0}$ (or $\hat{t}$ ) in the boundary term $\lambda e^{ \pm X^{0}}: X^{0} \rightarrow X^{0}+2 \pi i$. More generally, we have mixed thermal states due to $X^{0} \rightarrow X^{0}+2 n \pi i$, with positive integer $n$. Thus, the Hagedorn temperature $T_{H a g}^{\left(x^{0}\right)}=1 /(4 \pi)$ is just the case of $n=2$ for bosonic string theory. Similar mixed states for the host black hole can also be constructed from the periodicity of the imaginary time $x^{0}$.

For superstring theory, the obtained Hagedorn temperature is: $T_{H a g}^{\left(x^{0}\right)}=1 /(2 \sqrt{2} \pi)$, which arises from the periodicity: $X^{0} \rightarrow X^{0}+2 \sqrt{2} \pi i$ in the boundary term $\lambda e^{ \pm X^{0} / \sqrt{2}}$.

The temperatures for mixed states are: $T_{\text {Hag }}^{\left(x^{0}\right)}=1 /(2 \sqrt{2} n \pi)(n \geq 1)$. On the other hand, the Hawking temperature detected in the $x^{0}$ coordinate is also $1 /(2 \sqrt{2} \pi)$ from $x^{0} \rightarrow x^{0}+$ $2 \sqrt{2} \pi i$, as have been discussed in Section 2.2. So, once again, the Hagedorn temperature for open string creation on the probe brane is equal to the Hawking temperature of the host black brane (8):

$$
T_{H}=T_{\text {Hag }}^{(t)}=\frac{7-p}{\sqrt{2} r_{+}} \epsilon^{\frac{5-p}{2}} T_{\text {Hag }}^{\left(x^{0}\right)}, \quad T_{\text {Hag }}^{\left(x^{0}\right)}=\frac{1}{2 \sqrt{2} \pi} .
$$

It is worth pointing out that the equivalence between the two temperatures is independent of the specific construction of tachyon effective actions. All the temperatures actually arise from the unique periodicity of the Euclidian time coordinate in Rindler space. If we recover the factor $1 / \sqrt{\alpha^{\prime}}$ before $X^{0}$ in the boundary term, then there should be a corresponding $1 / \sqrt{\alpha^{\prime}}$ before $T$ in $V(T)$ in the effective theory (in this case, both $X^{0}$ and $T$ become of dimension length). Accordingly, the period of imaginary $x^{0}$ should be multiplied by a factor $\sqrt{\alpha^{\prime}}$. The resulting temperatures are still the same.

\subsection{Closed string emission: the increased entropy}

The Hagedorn temperature is the maximum temperature of a system and it usually signals phase transition. The energy of the rolling tachyon should be converted into closed string radiation due to the exponentially increasing open string mass. Since the Hagedorn temperature for open string pair creation on the unstable $q$-brane is equal to the Hawking temperature of the host black hole, the absorption and the emission of massless closed strings on the $q$-brane should be in equilibrium, with the same rate. So the emission of massless closed strings from the rolling tachyon discussed in [23, 24] should be suppressed. Actually, it has been found in [13, 14, that low dimensional unstable D-branes indeed decay 
into mainly very massive closed string states. On the other hand, closed strings can also split into open strings at the Hagedorn temperature [25].

In the leading order of $g_{s}$, the total average energy and number (per spatial worldvolume $V_{q}$ ) of emitted closed strings with large level number $n$ are calculated to be [14]:

$$
\frac{\bar{E}}{V_{q}}=(2 \pi)^{-q} \int d E E^{-\frac{q}{2}}, \quad \frac{\bar{N}}{V_{q}}=(2 \pi)^{-q} \int \frac{d n}{2 n}(4 n)^{-\frac{q}{4}},
$$

in either bosonic string or superstring theory. For an unstable D $q$-brane $(q \leq 2)$, the emitted energy diverges. We can choose the energy cut-off at the order of the mass or tension of the unstable particle or D $q$-brane. This means that the energy completely converts to the closed strings, with most energy transferred into the very massive states, towards the end of condensation. In the present context, this implies that the infalling wrapped $q$-brane $(q \leq 2)$ will dissolve into closed strings when reaching the horizon in infinitely long time as observed at infinity.

Let us first consider dropping a particle into the Schwarzschild black hole. On the macroscopic side in classical gravity, the black hole mass $M$ increases by $\delta M \sim m_{0}$. When entering into the near-horizon region, this particle behaves like a decaying unstable particle. On the microscopic side in the tachyon field theory or BCFT, the particle completely decays into closed strings, mainly massive modes, when it approaches the horizon. In terms of the first law, the black hole entropy $S$ should increase by $\delta S \sim \delta M / T_{H}$ due to capture of the particle (ignoring the charge and angular momentum contributions). This increased entropy should be accounted for by information stored in the closed strings that the particle decays into, as will be examined below.

Involving the relation (17), the first law then reads:

$$
\delta S \sim \frac{m_{0}}{T_{H}}=\frac{r_{0} m_{0}}{T_{H a g}^{\left(x^{0}\right)}} .
$$

This is the Hagedorn growth rule of entropy relying on mass commonly seen in string theory [26]. This equation says that the increased entropy should be the ratio of the corresponding mass $\left(m_{0}\right.$ or $\left.r_{0} m_{0}\right)$ of the test particle to the corresponding Hagedorn temperature $\left(T_{\text {Hag }}^{(t)}=T_{H}\right.$ or $\left.T_{H a g}^{\left(x^{0}\right)}\right)$ respectively observed in the $t$ or $x^{0}$ coordinate.

We now check whether the degenerate states of the emitted closed strings can account for the increased entropy in the host black hole required by the first law (20). The density of states of the emitted closed strings is obtained in [14] and it takes the form of the one of bosonic open strings in Hilbert space

$$
d_{n} \sim n^{-\frac{27}{4}} e^{4 \pi \sqrt{n}}
$$


with $E^{2} \sim 4 n$ at large level $n$. The energy $E$ is the order of the mass $r_{0} m_{0}$ of the particle measured in the $x^{0}$ coordinate. So the entropy contributed by the closed string states is

$$
\ln d_{n} \sim \frac{r_{0} m_{0}}{2 T_{\text {Hag }}^{\left(x^{0}\right)}}-\frac{27}{2} \ln \left(\frac{r_{0} m_{0}}{2}\right) .
$$

Thus, the leading term gives half of the result required by the first law in Eq. (20). The $1 / 2$ factor may arise from approximations, like the left-right mover identical assumption, adopted in [14] or in the above treatments. If we use the expression of closed string degenerate states in stead of the open string one (21) $: d_{n}^{(\text {clsoed })}=\left(d_{n}^{(\text {open })}\right)^{2}$, the result will agree with (20).

In the black brane backgrounds, the tension of the probe $q$-brane is dependent on the parameter $\epsilon$. At infinity, the tension is of order $\tau_{q} g_{s}^{-1}$. Approaching the near horizon region, the effective tension is $\tau_{q} g_{s}^{-1} \epsilon^{-\frac{(p-2)(7-p)}{4}}$. It can become very large or small towards the extremal limit, which means that there can be arbitrarily large or small amount of closed string radiated at the end of tachyon condensation. But, for a probe $q$-brane with given tension at infinity, the closed strings emitted from it should be limited. So there should be large deviation in the case $\epsilon \rightarrow 0$, in which there should be a different or revised theory to describe the dynamics. Therefore, we only consider the case away from extremality with $\epsilon \sim \mathcal{O}(1)$. In this case, the increased entropy in the black brane due to absorption of a wrapped $q$-brane can be expressed as, following the first law

$$
\delta S \sim \frac{\tau_{q} g_{s}^{-1}}{T_{H}} \sim \frac{2 T_{q}}{T_{H a g}^{\left(x^{0}\right)}} .
$$

where the tension $T_{q} \sim r_{+} g_{s}^{-1} \tau_{q}$ of the unstable $q$-brane is defined in Eq. (12). In superstring theory, the density of states should grow slower. If the degenerate state is still the open string one: $d_{n} \sim n^{-11 / 4} \exp (2 \pi \sqrt{2 n})$ [26], we shall also get half of the result (23).

\section{Conclusions}

The dynamics of infalling material in the near-horizon Rindler space can be described by the time-dependent tachyon field theory, in a holographic sense. Hence, the infalling material will experience a Hagedorn transition when falling through the near-horizon region of non-extremal black holes or branes. It will unavoidably dissolve into closed strings as approaching the horizon. This transition should be related to the confinementdeconfinement phase transition in holographic QCD models.

Our results have two important implications. One is that we should not automatically assume that any matter can move into the near-horizon region without any change in 
itself. As we show, the material will get thermalised and will decay into something else (closed strings) in this region. In plenty of previous classical discussions, this assumption has actually been adopted. Another implication of our results is that the information should be not lost when matter collapse into a black hole. The information is stored in the closed strings emitted from the particle. But here we do not transplant our discussion into the traditional semi-classical approach and find out the origin of information loss in there, which is left for further study.

\section{Acknowledgements}

We would like to thank Jianxin Lu for useful comments. 


\section{References}

[1] A. Strominger and C. Vafa, Microscopic Origin of the Bekenstein-Hawking Entropy, Phys. Lett. B379 (1996) 99-104, hep-th/9601029.

[2] C. G. Callan and J. M. Maldacena, D-brane Approach to Black Hole Quantum Mechanics, Nucl. Phys. B472 (1996) 591-610, hep-th/9602043.

[3] G. T. Horowitz and A. Strominger, Counting States of Near-Extremal Black Holes, Phys. Rev. Lett. 77 (1996) 2368-2371, hep-th/9602051.

[4] A. Dabholkar, Tachyon condensation and black hole entropy, Phys. Rev. Lett. 88 (2002) 091301, hep-th/0111004.

[5] G. T. Horowitz, Tachyon condensation and black strings, JHEP 08 (2005) 091, hep-th/0506166.

[6] S. W. Hawking, Breakdown of Predictability in Gravitational Collapse, Phys. Rev. D14 (1976) 2460-2473.

[7] A. Sen, Rolling Tachyon, JHEP 04 (2002) 048, hep-th/0203211.

[8] A. Sen, Tachyon matter, JHEP 07 (2002) 065, hep-th/0203265.

[9] A. Sen, Tachyon dynamics in open string theory, Int. J. Mod. Phys. A20 (2005) 5513-5656, hep-th/0410103.

[10] A. Strominger, Open string creation by S-branes, hep-th/0209090.

[11] M. Gutperle and A. Strominger, Timelike boundary Liouville theory, Phys. Rev. D67 (2003) 126002, hep-th/0301038.

[12] A. Maloney, A. Strominger, and X. Yin, S-brane thermodynamics, JHEP 10 (2003) 048, hep-th/0302146.

[13] T. Okuda and S. Sugimoto, Coupling of rolling tachyon to closed strings, Nucl. Phys. B647 (2002) 101-116, hep-th/0208196.

[14] N. D. Lambert, H. Liu, and J. M. Maldacena, Closed strings from decaying D-branes, JHEP 03 (2007) 014, hep-th/0303139.

[15] D. Kutasov and V. Niarchos, Tachyon effective actions in open string theory, Nucl. Phys. B666 (2003) 56-70, hep-th/0304045. 
[16] D. Kutasov, D-brane dynamics near NS5-branes, hep-th/0405058.

[17] D. Kutasov, A geometric interpretation of the open string tachyon, hep-th/0408073.

[18] D. N. Kabat and G. Lifschytz, Tachyons and black hole horizons in gauge theory, JHEP 12 (1998) 002, hep-th/9806214.

[19] A. Sen, Open and closed strings from unstable D-branes, Phys. Rev. D68 (2003) 106003, hep-th/0305011.

[20] G. T. Horowitz and A. Strominger, Black strings and P-branes, Nucl. Phys. B360 (1991) 197-209.

[21] M. J. Duff and J. X. Lu, Black and super p-branes in diverse dimensions, Nucl. Phys. B416 (1994) 301-334, hep-th/9306052.

[22] K. Okuyama, Wess-Zumino term in tachyon effective action, JHEP 05 (2003) 005, hep-th/0304108.

[23] B. Chen, M. Li, and F.-L. Lin, Gravitational radiation of rolling tachyon, JHEP 11 (2002) 050, hep-th/0209222.

[24] S.-J. Rey and S. Sugimoto, Rolling tachyon with electric and magnetic fields: Tduality approach, Phys. Rev. D67 (2003) 086008, hep-th/0301049.

[25] M. C. Batoni Abdalla, M. Botta Cantcheff, and D. L. Nedel, Strings in horizons, dissipation and a simple interpretation of the Hagedorn temperature, Eur. Phys. J. C54 (2008) 311-317, hep-th/0703064.

[26] M. B. Green, J. H. Schwarz, and E. Witten, SUPERSTRING THEORY. VOL. 1: INTRODUCTION, . Cambridge, Uk: Univ. Pr. ( 1987) 469 P. ( Cambridge Monographs On Mathematical Physics). 